\title{
Research Paper: Effect of Fatigue on Knee Kine- matics and Kinetics During Walking in Individuals With Flat Feet
}

\author{
Nader Farahpour ${ }^{1}$, Keyvan Sharifmoradi2* ${ }^{\circ}$, Saleh Azizi ${ }^{1}$ \\ 1. Department of Management and Motor Behavior, Faculty of Sport Sciences, Bu-Ali Sina University, Hamadan, Iran \\ 2. Department of Physical Education and Sport Sciences, School of Humanities, University of Kashan, Kashan, Iran.
}

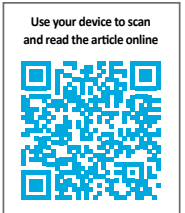

बittation Farahpour N, Sharifmoradi K, Azizi S. Effect of Fatigue on Knee Kinematics and Kinetics During Walking in Individuals With Flat Feet. Physical Treatments. 2017; 7(3):141-148. http://dx.doi.org/10.32598/ptj.7.3.141

: http://dx.doi.org/10.32598/ptj.7.3.141

Funding: See Page 146

(c) Copyright: The Author(s)

Article info:

Received: 10 Feb 2017

Accepted: 25 Jul 2017

Available Online: 01 Oct 2017

Keywords:

Flat foot, Fatigue, Knee, Kinetics, Gait

\section{A B S T R A C T}

Purpose: Flat feet associates with altered knee kinematics, kinetics, as well as knee pain. Fatigue of plantar intrinsic foot muscles may increase the navicular drop. However, it is unclear how fatigue influences the knee pain in individuals with flat feet. The purpose of this study was to assess the effect of fatigue on knee kinematics and kinetics in flat feet people during walking.

Methods: This is a quasi-experimental research. Ten individuals with flat feet (Mean $\pm \mathrm{SD}$ age: $24.4 \pm 2.16 \mathrm{y}$; Mean \pm SD height: $177.2 \pm 4.31 \mathrm{~cm}$; Mean \pm SD mass: $81.9 \pm 17.4 \mathrm{~kg}$ ) and 10 normal subjects (Mean \pm SD age: $25.28 \pm 6.33 \mathrm{y}$; Mean \pm SD height: $168.61 \pm 27.71 \mathrm{~cm}$; Mean \pm SD mass: $78.13 \pm 26.93 \mathrm{~kg}$ ) were participated in this study. A Vicon motion analysis system $(100 \mathrm{~Hz})$ with four cameras and two Kistler force plates $(1000 \mathrm{~Hz})$ were used to measure knee kinematics and kinetics during gait before and after fatigue. For between group and within group comparisons, the Independent $t$ test and repeated measures analysis of variance were used, respectively. SPSS (V. 22) was used to analyze data with the significance level of $\mathrm{P}<0.05$.

Results: Knee range of motion of flat feet group in frontal plane ( $9.37 \pm 2.54$ degree) was significantly higher than that of healthy group $(\mathrm{P}=0.01)$ in the pretest. In the flat feet group, the knee moment in sagittal, frontal, and horizontal plan was significantly greater than those in the healthy group by $0.86(\mathrm{P}=0.002), 0.25(\mathrm{P}=0.016)$ and $0.19(\mathrm{P}=0.000) \mathrm{Nm} / \mathrm{BW}$, respectively in the pretest. Adductor moment increased after fatigue protocol in the healthy group by $0.08 \mathrm{Nm} / \mathrm{BW}$.

Conclusion: Before exhaustion, the knee moments in sagittal, frontal, and horizontal planes in the flat feet group were significantly higher than that in the healthy group. In the flat feet group, fatigue resulted in a decrease on the knee flexion and abduction moments and increase in knee ROM in sagittal and frontal plane in the flat foot group. The decreased knee muscle moments may result in an increased loading on the knee joint. It appears that extreme exhausting activity might place the knees of flat feet individuals at the risk of pathology and injury.

\footnotetext{
* Corresponding Author:

Keyvan Sharifmoradi, $P h D$

Address: Department of Physical Education and Sport Sciences, School of Humanities, University of Kashan, Kashan, Iran.

Phone: +98 (31) 55913707

E-mail: ksharifmoradi@gmail.com
} 


\section{Introduction}

F

lat feet is common in children with the prevalence of $35 \%$ in boys and $20 \%$ in girls [1]. It is less prevalent in older ages since it becomes corrected by age [2-4]. Foot pronation is a risk factor for mechanical dysfunctions and injuries in the upper joints such as ankle, knee and hip joints $[5,6]$. In theory, foot pronation creates tibia internal rotation, hip internal rotation [7], knee valgus and pelvis anterior and ipsilateral tilt [6].

In knee valgus, more axial loads are imposed on the lateral compartment of knee [7] which may be a source of the knee pain and discomfort. Generally, the amplitude of navicular drop increases with fatigue. Fatigue refers to a reduction in the efficiency and force generating capacity of muscles after prolonged exposure to activity [8]. Research has shown that fatigue can change kinetics and kinematics of the lower limbs in gait [9-12]. Yoshine et al. assessed the effect of prolonged walking fatigue on gait and found local muscle fatigue at tibialis anterior muscle, gait instability rhythm, and slow gait rhythm [9]. Barbieri et al. analyzed the effect of muscle fatigue on the kinematic and kinetic parameters of normal gait and found that fatigue would affect kinematic and kinetic parameters of gait [10].

Qu et al. examined the effects of load and fatigue on gait characteristics and found increased gait width variability, hip ROM, and trunk ROM with fatigue and load [11]. Hamacher et al. assessed the gait patterns in healthy young individuals by maximal fatigued and in older adults by submaximal fatigued. They found that young adult subjects become more stable after maximal fatigue but older individuals walk less locally stable in a submaximal fatigued condition [12]. Parijat et al. examined the effect of lower extremity fatigue on gait variables of healthy young adults. They found a significant decrease in the center of mass and peak knee joint moment and an increased knee flexion and reduced ankle dorsiflexion at the heel strike phase of the gait cycle during fatigue trials [13].

Long pre et al. found no changes in knee adduction moment, knee flexion angles, dynamic knee stiffness, and muscle co-activation after lower limb fatigue. They just found that the peak knee extension moment reduced during fatigue [14]. Hunt et al. assessed the effect of ankle plantar flexor fatigue on knee and ankle biomechanics during gait. They found out that fatigue significantly decreased medial gastrocnemius activity, and increased knee flexion ROM and external knee flexion moment [15].
Flat feet people experience pain and discomfort in knee joint while walking. However, to the best of authors' knowledge, so few published studies have assessed the effect of fatigue on knee joint kinematics and kinetics during gait in flat feet people. This study aimed to assess the effects of fatigue on knee joint kinematics and kinetics during walking. It was hypothesized that $\mathrm{A}$. Knee joint moment increases after fatigue, and B. Knee joint ROM increases after fatigue.

\section{Materials and Methods}

This is a quasi-experimental research. A group of 10 healthy individuals (Mean \pm SD age: $25.28 \pm 6.33$ y; Mean \pm SD height: $168.6 \pm 27.71 \mathrm{~cm}$; Mean \pm SD mass: $78.13 \pm 26.93 \mathrm{~kg}$ ) were selected as healthy group. Also, a group of 10 flat feet individuals (Mean \pm SD age: $24.41 \pm 2.63 \mathrm{y} ; \quad$ Mean \pm SD height; $177.26 \pm 4.33 \mathrm{~cm}$; Mean \pm SD mass: $33.61 \pm 2.93 \mathrm{~kg}$ ) were selected as experimental group. Subjects would be included in the experimental group if they did not have any history of major surgery, leg length discrepancy, pain, neuromuscular disease, or known balance deficit. The research protocol was approved by the Ethics Committee of Bu-Ali Sina University of Hamadan. Objectives and methods were explained for subjects and they gave their written consent before the experimentation.

Footprint method was used to quantify the foot arch. In this method, the footprint area was recorded by industrial carbon powder on A4 paper, and then the footprint was scanned and imported to Auto CAD software environment (Figure 1). Then, the length of the footprint was divided into three equal parts, and the area of the three parts was calculated separately. The arc-index of the foot was calculated using Equation 1 [16].

$$
\text { (1) } A I=\frac{B}{(A+B+C)} \times 100
$$

Where $\mathrm{AI}$ is the foot archindex, $\mathrm{A}, \mathrm{B}$ and $\mathrm{C}$ correspond to the front, middle and the rear one-third of the foot print length, respectively. This method has a moderate to high correlation with radiography methods [17-20]. Based on this foot print index, the foot can be classified into normal $(0.21>\mathrm{AI}<0.26)$, pes cavus $(\mathrm{AI}<0.21)$, and flat feet $(\mathrm{AI}>0.26)$ [21].

\section{Instruments}

A Vicon motion analysis system with four T-series cameras $(100 \mathrm{~Hz})$, with Nexus-1.7.5 software (Oxford metrics, UK), was used to shoot the movements of 16 reflective spherical markers attached to the lower limbs 


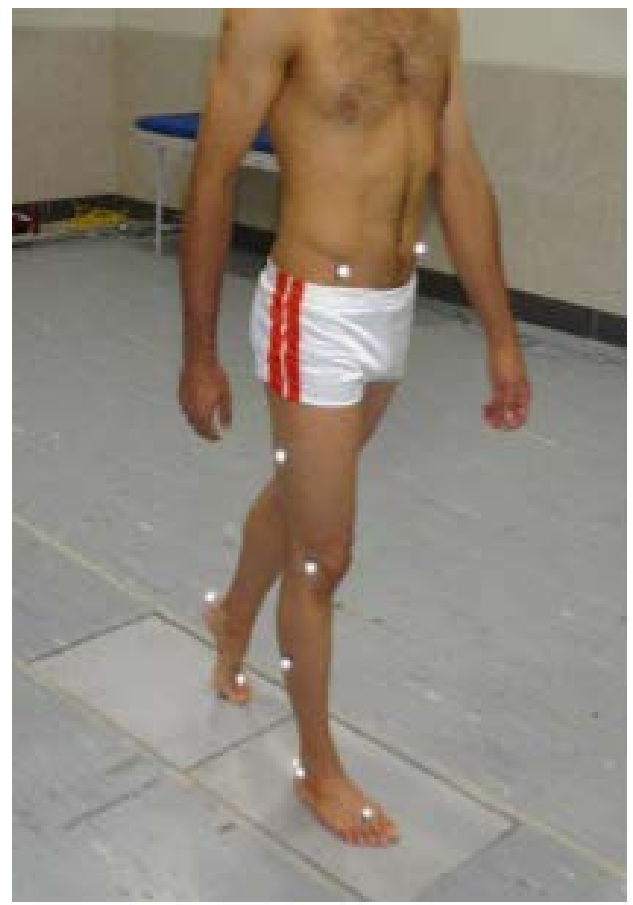

Figure 1. Marker placement

PHYSICAL TREA MENTS

during barefoot walking at self-selected speed. Based on plug-in-gait model, reflective markers were placed on bony landmarks over the Anterior Superior Iliac Spine (ASIS), Posterior Superior Iliac Spine (PSIS), Mid-Thigh (MT), Lateral Condyle of Femur (LCOF), Mid-Shank on the lateral side (MSH), Lateral Malleolus (LMA), heel, and toe over the distal head of the second metatarsal bilaterally (Figure 1).

Kinematic data were filtered using low-pass Butterworth forth-order filter with a cut-off frequency of $10 \mathrm{~Hz}$. Threedimensional range of motion for the right and left knee joints were measured. Two Kistler force plates were also used to record the Ground Reaction Forces (GRF) during gait. GRF data were sampled at $1000 \mathrm{~Hz}$. Force plates and the motion capture systems were synchronized. The protocol were implemented on both groups.

\section{Movement task}

The subjects were asked to walk with a self-selected speed for 5 successful trials. The relevant data were measured from right and left foot. A trial was defined successful if all markers were recorded throughout a stride. Then, after a fatigue protocol, another set of 5 successful walking trials were performed. The mean values of the 5 trials were used for each required parameter.

\section{Fatigue protocol}

The subjects were initially asked to warm-up for $5 \mathrm{~min}$ utes, including free walking and flexibility stretches. The fatigue protocol includes running until exhaustion. Then the subjects ran around an indoor volleyball salon $(90 \mathrm{~m}$ environment) with $4.5 \mathrm{~m} / \mathrm{s}$ speed for more than $12 \mathrm{~min}$ utes. If the exhaustion occurred before 12 minutes, the subject would be excluded from the study. After exhaustion, the test was stopped. Exhaustion was defined as being unable to continue running and a score of greater than 17 based on borg Rating of Perceived Exertion (RPE) scale [22]. Kinematics and kinetics data were recorded before and after the fatigue protocol.

\section{Data processing and statistics}

First using Nexus software, the kinematics and GRF data were processed and saved with c3d extension. Then data were imported into Visual 3D software. Knee moments and ROM in sagittal, frontal and horizontal plane were extracted from the visual 3D software (C-motion USA). Knee moments were normalized to body weight. Normal distribution of data was done by KolmogorovSimonov test to evaluate normality of data. The Independent $t$ test was used to compare between group differences on different variables at pretest. Repeated measures analysis of variance test was also used to show the interaction between factors and groups. Pretest data and plane (sagittal, frontal, and horizontal) were considered as a factor. Statistical significance was set at $\mathrm{P}<0.05$ for all statistical analysis.

\section{Results}

Table 1 summarizes the differences between flat feet and healthy groups regarding the knee ROM in sagittal, frontal and horizontal planes. The knee abduction in flat feet group was significantly greater by $32 \%$ than that in healthy group $(\mathrm{P}<0.01)$. The knee flexion and rotation were not statistically different between two groups $(\mathrm{P}>0.05)$. Figure 2 shows the significant interaction between plane and fatigue factors $\operatorname{ROM}(\mathrm{P}=0.044)$. According to the Figure 2, there was no significant difference in knee ROM in sagittal and horizontal plane between before and after fatigue protocol, but knee ROM in frontal plane was more at post-test compared to pretest. Knee ROM in frontal plane increased significantly 1.2 degrees at post-test.

The mean differences of knee moment between two groups before fatigue protocol are shown in Table 2 According to this table, sagittal and horizontal knee moment 
Table 1. Knee ROM (Mean \pm SD) between flat feet and healthy groups before fatigue (degrees)

\begin{tabular}{|c|c|c|c|c|c|}
\hline \multirow{2}{*}{ Side } & \multirow{2}{*}{ ROM } & \multicolumn{2}{|c|}{ Mean \pm SD } & \multirow{2}{*}{$\mathbf{P}$} & \multirow{2}{*}{ Sig. } \\
\hline & & Flat Feet & Healthy & & \\
\hline \multirow{3}{*}{ Right knee } & Flexion & $59.45 \pm 5.64$ & $62.24 \pm 1.72$ & -1.499 & 0.15 \\
\hline & Abduction & $9.37 \pm 2.54$ & $6.38 \pm 1.41$ & 3.265 & $0.01 *$ \\
\hline & Rotation & $3.74 \pm 1.90$ & $3.06 \pm 1.02$ & 0.991 & 0.33 \\
\hline \multirow{3}{*}{ Left knee } & Flexion & $61.54 \pm 2.85$ & $62.04 \pm 1.78$ & -0.477 & 0.64 \\
\hline & Abduction & $9.78 \pm 1.39$ & $9.80 \pm 2.76$ & -0.019 & 0.99 \\
\hline & Rotation & $3.88 \pm 1.34$ & $3.09 \pm 0.51$ & 1.750 & 0.10 \\
\hline
\end{tabular}

was significantly greater in flat feet group compared to healthy group on the right and left side. Moreover the first and second peak of adduction knee moment in frontal plane were significantly greater in flat feet group on the left side. Figure 3 displays the interaction of knee adduction moment between groups and fatigue. According to Figure 3, before fatigue protocol knee moment adductor of healthy group was significantly lower than that of flat feet group, but after fatigue protocol knee adductor moment did not change significantly between two groups. Adductor moment increased $0.08 \mathrm{Nm} / \mathrm{BW}$ after fatigue protocol in the healthy group.

\section{Discussion}

This study aimed to assess the effect of fatigue on knee moment kinematics and kinetics in flat feet people during walking. Our study results showed that knee ROM of the flatfeet group in frontal plane was significantly higher than that of the healthy group $(\mathrm{P}=0.00)$. In the flatfeet group, the knee moment in sagittal, frontal and horizontal plan was significantly greater by $0.86,0.02$ and $0.13 \mathrm{Nm} / \mathrm{BW}$, respectively.

The results showed that the knee adduction ROM in the flat feet group was significantly higher than that of the healthy group. The flat feet group walked with excessive pronation and hind foot eversion. The excessive pronation of the foot was accompanied by an increased leg and thigh internal rotation [23, 24]. Hind foot eversion can also lead to increase knee ROM in frontal plan [25]. So these factors can increase knee joint motion in frontal plan. Based on the results, knee motion in frontal plane increased significantly in flatfeet group compared to the healthy group at post-test.

In this study, there was no significant difference in sagittal and horizontal planes of knee ROM before fatigue protocol.

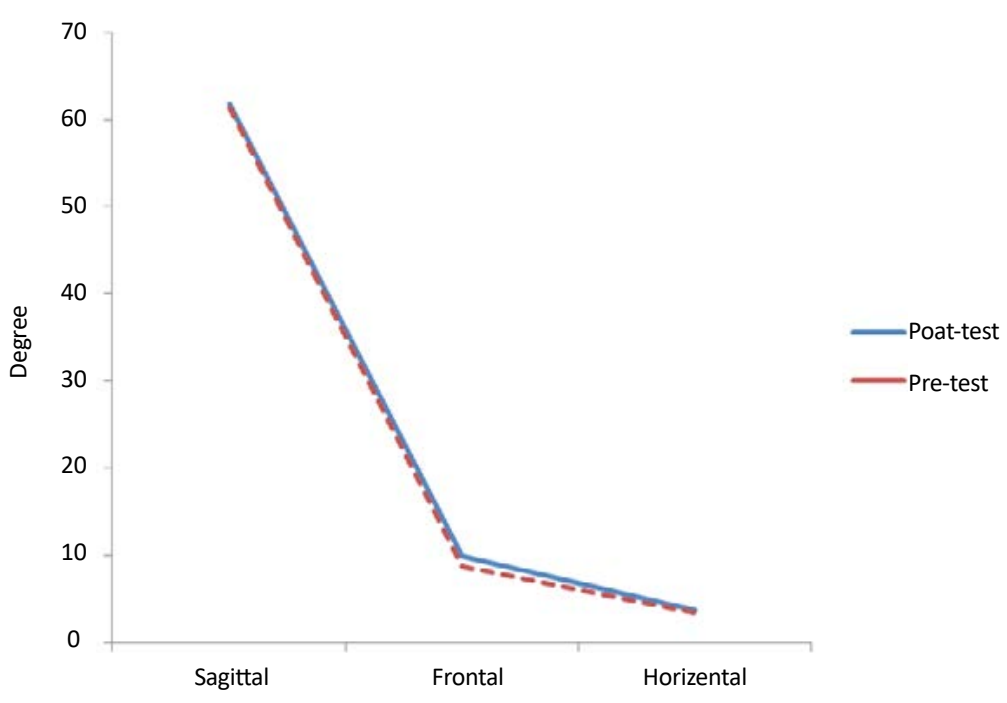

Figure 2. The interaction between plane and fatigue ROM 
Table 2. The mean differences of knee moment between two groups (Nm/BW) before fatigue protocol (t-test results)

\begin{tabular}{|c|c|c|c|c|}
\hline Side & Plane & Flat Feet (Mean $\pm S D$ ) & Healthy (Mean $\pm S D$ ) & Sig. \\
\hline \multirow{5}{*}{ Left } & Sagittal & $1.46 \pm 0.55$ & $0.71 \pm 0.28$ & $0.002^{*}$ \\
\hline & First peak frontal & $1.19 \pm 0.28$ & $0.94 \pm 0.06$ & $0.016^{*}$ \\
\hline & Valley frontal & $0.69 \pm 0.18$ & $0.52 \pm 0.19$ & 0.069 \\
\hline & Second peak frontal & $1.20 \pm 0.26$ & $0.94 \pm 0.11$ & $0.013^{*}$ \\
\hline & Horizontal & $0.29 \pm 0.05$ & $0.10 \pm 0.02$ & $0.001 *$ \\
\hline \multirow{5}{*}{ Right } & Sagittal & $1.48 \pm 0.65$ & $0.61 \pm 0.29$ & $0.002^{*}$ \\
\hline & First peak frontal & $0.45 \pm 0.08$ & $0.54 \pm 0.10$ & 0.062 \\
\hline & Valley frontal & $0.21 \pm 0.02$ & $0.20 \pm 0.10$ & 0.686 \\
\hline & Second peak frontal & $0.41 \pm 0.03$ & $0.45 \pm 0.17$ & 0.458 \\
\hline & Horizontal & $0.23 \pm 0.04$ & $0.05 \pm 0.03$ & $0.001^{*}$ \\
\hline
\end{tabular}

*: Significant difference between flat feet and healthy groups in both right and left feet

PHYSICAL TREA $\mid$ MENTS

Bultd et al. also reported no significant difference in the knee extension joint angle between the healthy and flatfeet groups, which was in line with the results of this study [26]. Although they observed more external rotation in the flatfeet group compared to the normal group, which was contradicted with the results of this study.

The results also showed that before fatigue protocol, extensor, adductor, and internal rotator moment of the flat feet group were significantly higher than that of the healthy group, that matches with the study results of Buldt and associates [26]. They showed that the knee joint adductor moment in the flat feet group was significantly higher than that of the healthy group [26].
The knee ROM in the frontal plane was significantly higher in the flat feet group compared to the healthy group. Increasing the range of knee joint motion in the frontal plane, along with increased knee joint adduction moment, can increase the pressure on the medial compartment of the knee joint and lead to increased cumulative forces participating in the destruction of knee articular cartilage. Although fewer studies have been conducted on the knee joint internal rotation ROM in the flat feet group, it is generally believed that rotational changes in the knee joint and applied load on knee cartilage are the basis for the onset of knee joint arthritis [27].

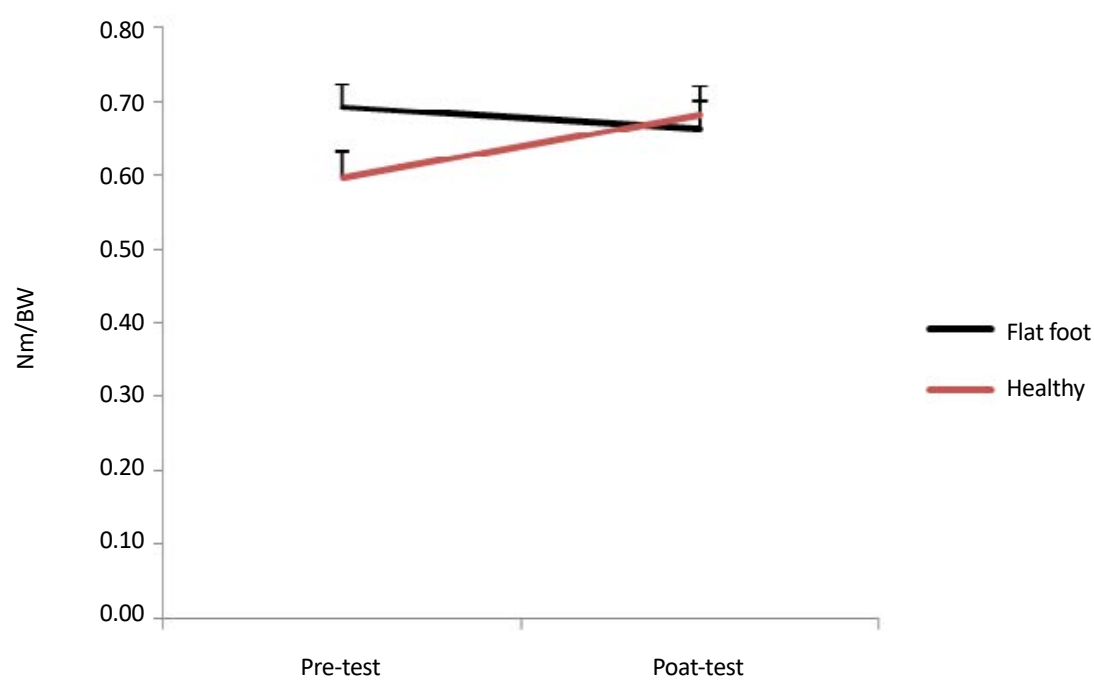

Figure 3. The interaction of knee moment adduction between group and fatigue 
Landry et al. reported an increased external rotational moment in individuals with knee osteoarthritis [28]. Mundermann et al. (2000) reported that increased knee adduction moment applied load on the medial compartment of the knee and possibly the change in rotational and adductor moment changes knee screw-home mechanism and can increase the risk of arthritic injury in flat feet group [29].

After fatigue, the knee joint ROM was higher in the frontal and horizontal planes of the flat feet group compared to the healthy group. On the contrary, knee joint moment of the flat feet group decreased, while the knee joint muscle moment of the healthy group increased after fatigue. Before the fatigue, the flat feet group showed increased muscle moment compared to the healthy group. Running with higher muscle moment while performing the fatigue protocol, may increase fatigue in the flat feet group more than that of the healthy group, so the flat feet group produced less moment at the end of the post-test compared to the healthy group because of more fatigue in their muscles.

Reducing muscle moment can increase knee joint ROM. The result of this study showed an increase in frontal and horizontal knee ROM after fatigue protocol in the flat feet group. Murdock et al. found that a high intensity quadriceps fatigue protocol resulted in decreased knee external rotation moment and increased external rotation angle. After fatigue, changes in muscle activation patterns were consistent with the biomechanical changes [30]. Parijat et al. examined the effect of lower extremity fatigue on gait variables of the healthy young adults. They found a significant decrease in peak knee joint moment during fatigue trials [13].

Other researchers showed that the activity of hamstring and quadriceps muscles would decrease after fatigue, due to the reduction of the knee moment, change in the muscle force production and the pattern of the muscle activity after fatigue. These mechanical changes reduce stabilizer forces with fatigue induced on the knee [30], so lower muscle activity and changes in muscle forces production pattern after fatigue will expose more knee joints to cumulative loads.

The knee moments in all planes were significantly higher in the flat feet group compared to the healthy group in the pretest. Knee flexion and abduction moments decrease in the flat feet group due to fatigue and knee ROM increase in sagittal and frontal plane. The decreased knee muscle moments may result in an increased loading on the knee joint. It appears that the knee of flat feet individuals might be at the risk of pathology and injury at extreme exhausting activities.

\section{Ethical Considerations}

\section{Compliance with ethical guidelines}

The research protocol was approved by the Ethics Committee of Bu-Ali Sina University of Hamadan. Objectives and methods were explained for subjects and they gave their written consent before the experimentation.

\section{Funding}

This research did not receive any specific grant from funding agencies in the public, commercial, or not-forprofit sectors.

\section{Conflict of interest}

The authors declared no conflict of interest.

\section{Acknowledgements}

The authors would like to express their sincere appreciation to all who participated in this study.

\section{References}

[1] Chen JP, Chung MJ, Wang MJ. Flatfoot prevalence and foot dimensions of 5- to 13- Year Old Children in Taiwan. Foot \& Ankle International. 2009; 30(4):326-32. [DOI:10.3113/ FAI.2009.0326] [PMID]

[2] Burns J, Keenan AM, Redmond A. Foot type and overuse injury in triathletes. Journal of the American Podiatric Medical Association. 2005; 95(3):235-41. [DOI:10.7547/0950235] [PMID]

[3] Barnes A, Wheat I, Milner C. Association between foot type and tibial stress injuries: A systematic review. British Journal of Sports Medicine. 2008; 42(2):93-8. [DOI:10.1136/ bjsm.2007.036533] [PMID]

[4] Kosashvili Y, Fridman T, Backstein D, Safir O, Ziv YB. The correlation between pes planus and anterior knee or intermittent low back pain. Foot \& Ankle International. 2008; 29(9):910-3. [DOI:10.3113/FAI.2008.0910] [PMID]

[5] Harradine P, Bevan L, Carter N. An overview of podiatric biomechanics theory and its relation to selected gait dysfunction. Physiotherapy. 2006; 92(2):122-7. [DOI:10.1016/j.physio.2005.10.003]

[6] Bird A, Payne C. Foot function and low back pain. The Foot 1999; 9(4):175-80. [DOI:10.1054/foot.1999.0563]

[7] Tiberio D. The effect of excessive subtalar joint pronation on patellofemoral mechanics: A theoretical model. Journal 
of Orthopaedic \& Sports Physical Therapy. 1987; 9(4):160-5. [DOI:10.2519/jospt.1987.9.4.160]

[8] Gandevia SC, Allen GM, McKenzie DK. Central fatigue. In: Cohen IR, Lajjtha A, Lambris JD, Paoletti R, Rezaei $\mathrm{N}$, editors. Advances in Experimental Medicine and Biology. Cambridge: New \& Forthcoming Titles; 1967. [DOI:10.1007/978-1-4899-1016-5]

[9] Yoshino K, Motoshige T, Araki T, Matsuoka K. Effect of prolonged free-walking fatigue on gait and physiological rhythm. Journal of Biomechanics. 2004; 37(8):1271-80 [DOI:10.1016/j.jbiomech.2003.11.031] [PMID]

[10] Barbieri FA, dos Santos PCR, Vitório R, van Dieën JH, Gobbi LTB. Effect of muscle fatigue and physical activity level in motor control of the gait of young adults. Gait \& Posture. 2013; 38(4):702-7. [DOI:10.1016/j.gaitpost.2013.03.006] [PMID]

[11] Qu X, Yeo JC. Effects of load carriage and fatigue on gait characteristics. Journal of Biomechanics. 2011; 44(7):1259-63. [DOI:10.1016/j.jbiomech.2011.02.016] [PMID]

[12] Hamacher D, Törpel A, Hamacher D, Schega L. The effect of physical exhaustion on gait stability in young and older individuals. Gait \& Posture. 2016; 48:137-9. [DOI:10.1016/j. gaitpost.2016.05.007] [PMID]

[13] Parijat P, Lockhart TE. Effects of quadriceps fatigue on the biomechanics of gait and slip propensity. Gait \& Posture. 2008; 28(4):568-73. [DOI:10.1016/j.gaitpost.2008.04.001] [PMID] [PMCID]

[14] Longpré HS, Potvin JR, Maly MR. Biomechanical changes at the knee after lower limb fatigue in healthy young women. Clinical Biomechanics. 2013; 28(4):441-7. [DOI:10.1016/j. clinbiomech.2013.02.010] [PMID]

[15] Hunt MA, Hatfield GL. Ankle and knee biomechanics during normal walking following ankle plantarflexor fatigue. Journal of Electromyography and Kinesiology. 2017; 35:249. [DOI:10.1016/j.jelekin.2017.05.007] [PMID]

[16] Bertani MC. Differences of foot arch index and plantar pressure in elderly people during standing: Considering gender, age and foot dominance [MSc. thesis]. Porto: University of Porto; 2014.

[17] Menz HB. Alternative techniques for the clinical assessment of foot pronation. Journal of the American Podiatric Medical Association. 1998; 88(3):119-29. [DOI:10.7547/87507315-88-3-119] [PMID]

[18] Saltzman CL, Nawoczenski DA, Talbot KD. Measurement of the medial longitudinal arch. Archives of Physical medicine and Rehabilitation. 1995; 76(1):45-9. [DOI:10.1016/ S0003-9993(95)80041-7]

[19] McCrory J, Young M, Boulton A, Cavanagh P. Arch index as a predictor of arch height. The Foot. 1997; 7(2):79-81. [DOI:10.1016/S0958-2592(97)90052-3]

[20] Menz HB, Munteanu SE. Validity of 3 clinical techniques for the measurement of static foot posture in older people. Journal of Orthopaedic \& Sports Physical Therapy. 2005; 35(8):479-86. [DOI:10.2519/jospt.2005.35.8.479] [PMID]

[21] Murley GS, Menz HB, Landorf KB. Foot posture influences the electromyographic activity of selected lower limb muscles during gait. Journal of Foot and Ankle Research. 2009; 2(1):35. [DOI:10.1186/1757-1146-2-35] [PMID] [PMCID]
[22] Borg G. Borg's perceived exertion and pain scales. Champaign, Illinois: Human Kinetics; 1998.

[23] Tiberio D. Pathomechanics of structural foot deformities. Physical Therapy. 1988; 68(12):1840-9. [DOI:10.1093/ $\mathrm{ptj} / 68.12 .1840]$ [PMID]

[24] Dierks TA, Manal KT, Hamill J, Davis IS. Proximal and distal influences on hip and knee kinematics in runners with patellofemoral pain during a prolonged run. Journal of Orthopaedic \& Sports Physical Therapy. 2008; 38(8):448-56. [DOI:10.2519/jospt.2008.2490] [PMID]

[25] Williams DS, McClay IS, Hamill J, Buchanan TS. Lower extremity kinematic and kinetic differences in runners with high and low arches. Journal of Applied Biomechanics. 2001; 17(2):153-63. [DOI:10.1123/jab.17.2.153]

[26] Buldt AK, Levinger P, Murley GS, Menz HB, Nester CJ, Landorf KB. Foot posture and function have only minor effects on knee function during barefoot walking in healthy individuals. Clinical Biomechanics. 2015; 30(5):431-7. [DOI:10.1016/j.clinbiomech.2015.03.014] [PMID]

[27] Andriacchi TP, Briant PL, Bevill SL, Koo S. Rotational changes at the knee after ACL injury cause cartilage thinning. Clinical Orthopaedics and Related Research. 2006; 442(442):39-44. [DOI:10.1097/01.blo.0000197079.26600.09] [PMID]

[28] Landry SC, McKean KA, Hubley-Kozey CL, Stanish WD, Deluzio KJ. Knee biomechanics of moderate OA patients measured during gait at a self-selected and fast walking speed. Journal of Biomechanics. 2007; 40(8):1754-61. [DOI:10.1016/j.jbiomech.2006.08.010] [PMID]

[29] Mündermann A, Dyrby CO, Andriacchi TP. Secondary gait changes in patients with medial compartment knee osteoarthritis: increased load at the ankle, knee, and hip during walking. Arthritis \& Rheumatology. 2005; 52(9):2835-44. [DOI:10.1002/art.21262] [PMID]

[30] Murdock GH, Hubley-Kozey CL. Effect of a high intensity quadriceps fatigue protocol on knee joint mechanics and muscle activation during gait in young adults. European journal of Applied Physiology. 2012; 112(2):439-49. [DOI:10.1007/s00421-011-1990-4] [PMID] 
